\title{
Una investigación-acción sobre tiempo histórico
}

\author{
Joan Llusà Serra ${ }^{1}$ \\ Recibido: 20 de septiembre de 2016 - Aceptado: 14 de octubre de 2016
}

\begin{abstract}
Resumen
Este artículo se divide en cuatro apartados. En primer lugar, encontramos la introducción, donde se justifica la importancia de aplicar de forma sistemática al proceso de enseñanza y aprendizaje de la historia el concepto temporal de la contemporaneidad. En segundo lugar, se describe el proceso de investigación desarrollado, incluyendo modelo, metodología, instrumentos y contexto. Se trata de un modelo de investigación-acción de didáctica de la historia que, como estudio de caso, pretende dar respuesta al problema de la comprensión del tiempo histórico por parte de mi propio alumnado. En tercer lugar, se presentan los resultados de la investigación y, finalmente, se establecen unas conclusiones, destacando la importancia de la enseñanza y del aprendizaje del tiempo histórico y específicamente de la contemporaneidad, como base para el desarrollo de una historia más comprensiva, funcional y capaz de romper con el eurocentrismo tradicional para sentar las bases de una historia global.
\end{abstract}

Palabras Clave: Contemporaneidad, Tiempo Histórico, Historia comprensiva, Didáctica de la historia.

\section{An Action-Research on Historical Time}

\begin{abstract}
This article is divided into four sections. In the first place, we find the introduction, which justifies the importance of systematically applying to the teaching and learning process of history, the temporary concept of contemporaneity. Secondly, it describes the research process developed, including model, methodology, tools and context. It is an action-research model of teaching approach of history that, as a case study, aims to give an answer to the problem of understanding of historical time by my own students. Thirdly, the results of the research are presented and, finally, conclusions are drawn, highlighting the importance of teaching and learning of historical time and specifically of contemporaneity, as a basis for the development of a more comprehensive
\end{abstract}

1 Español. Doctor en Didáctica de las Ciencias Sociales. Académico, Universidad Autónoma de Barcelona, España. E-mail: juan.llusa@uab.cat 
and functional history, capable of breaking the traditional Eurocentrism and lay the foundations of a global history.

Keywords: Contemporaneity, Historical Time, Comprehensive History, Teaching Approach of history

\begin{abstract}
Uma investigação-ação sobre tempo histórico Resumo

Este artigo está dividido em quatro seções. No primeiro, encontramos a introdução, onde se justifica a importância de aplicar sistematicamente o processo de ensino e aprendizagem da história o conceito temporal da contemporaneidade. Em segundo lugar, descreve-se o processo de pesquisa desenvolvida, incluindo modelo, metodologia, instrumentos e contexto. Trata-se de um modelo do método investigação- ação de didática da história que, como estudo de caso, pretende dar resposta ao problema da compreensão do tempo histórico por parte dos meus próprios alunos. Em terceiro lugar, se apresentam os resultados da pesquisa e, finalmente, se estabelecem as conclusões, destacando a importância do ensino e da aprendizagem do tempo histórico e, especificamente, da contemporaneidade, como base para o desenvolvimento de uma história mais compreensiva, funcional e capaz de romper com o euro-centrismo tradicional para assentar as bases de uma história global.
\end{abstract}

Palavras-chave: Contemporaneidade, Tempo Histórico, História Compreensiva, Didática da história.

Stenhouse (1998) afirmaba que un día serán los profesores quienes en definitiva cambiarán el mundo de la escuela entendiéndola. Pero ¿cómo puede un profesor entender la escuela?, ¿Cómo puede entender su escuela, su praxis, su realidad cotidiana? La respuesta es ciertamente compleja, pero a mi entender pasa inevitablemente por la investigación-acción. Efectivamente, los maestros y maestras del siglo XXI definitivamente deben dar un paso adelante para cambiar el proceso de enseñanza y aprendizaje de la historia y, por ello, el modelo del profesional reflexivo como transformador de realidades educativas tiene que convertirse en el modelo a seguir.

Es precisamente desde este paradigma donde nace la necesidad de investigar mi propia práctica. Como profesor de secundaria, la observación diaria de mis clases y la constatación de las dificultades del alumnado por comprender realmente la historia y no solo memorizarla, y, a la vez, mi incapacidad para encontrar estrategias didácticas eficaces para otorgarle una mayor funcionalidad y utilidad, me llevaron a un análisis detallado de las causas profundas que dificultaban la consecución de este doble objetivo. A pesar de que las causas son múltiples: la existencia de un currículum demasiado rígido e ina- 
bordable, una estructura escolar desfasada, la preponderancia de un modelo tradicional de docencia, el uso de materiales y recursos poco adecuados, a mi entender, el eje central en torno al cual pivota un aprendizaje de la historia realmente comprensivo y competencial es el dominio del tiempo histórico y de los conceptos que lo articulan.

Sin embargo, la constatación cotidiana de las enormes dificultades de los chicos y chicas de secundaria por entender y aplicar adecuadamente los distintos operadores temporales, me llevaron al desarrollo de un primer trabajo de investigación y a la posterior tesis doctoral, con el objetivo de dar respuesta a estas dificultades y encontrar nuevas vías para aplicar en mi praxis modelos más eficaces de enseñanza y aprendizaje de la historia.

Todas las investigaciones, como las recogidas por Pagès (1999): González (1993), Grupo de Valladolid (1994), González García (1995), Teruel (1998), Gil et al. (1997), Alonso Tapia (1997) o las de Barton y Lewstik (1996), Lautier (1997), confirman la enorme importancia que otorga el profesorado al tiempo histórico para enseñar historia, pero a la vez también la poca aplicación real en sus clases. La justificación para explicar esta situación recae principalmente en las dificultades de comprensión y aplicación del mismo por parte del alumnado.

En este sentido, por ejemplo, Lautier (1997: 2) afirma "Lorsqu'ils parlent du temps, les enseignants libèrent leurs inquiétudes sur les capacités limitées de leurs élèves".

Por ello, Pagès afirma:

"La enseñanza y el aprendizaje de la historia no responde a aquello que los jóvenes esperan encontrar ni a aquello que algunos creemos que deberían encontrar para orientarse en su mundo, para desarrollar su temporalidad, para formar su conciencia histórica. Los saberes históricos escolares no tienen para el alumno ningún sentido más allá de las paredes de la escuela." (2003: 2).

Estas dificultades observadas y contrastadas en mis propias aulas, me llevaron a la construcción de un modelo de enseñanza y aprendizaje de la historia basado en la contemporaneidad. En Italia, el término contemporaneidad es sinónimo de simultaneidad pero, en este caso, si bien la contemporaneidad nace de la sincronía y se puede relacionar con este concepto, va más allá de la simple coincidencia temporal.

La contemporaneidad pretende, a partir de la identificación de acontecimientos simultáneos y el establecimiento de relaciones entre ellos, poder explicar holísticamente cualquier realidad histórica y finalmente tener la competencia de aplicar este modelo a situaciones o contextos diversos. Se trata pues de un concepto temporal con distintos niveles de complejidad 
creciente, basados en la Taxonomía de Bloom (1957 y 1971) y revisada posteriormente por Anderson (2000). Estos 4 niveles de complejidad creciente en las que se divide la contemporaneidad: identificar, relacionar, explicar y aplicar a contextos múltiples, además, revisten de mayor o menor dificultad dependiendo de la proximidad temporal y territorial desde donde se realiza el análisis. Finalmente, también se vincula con el modelo de historia global defendida por McNeil (2004) o Christian (2005) y con la formación por competencias, es decir, con una forma de entender la enseñanza y el aprendizaje de la historia vinculada a la comprensión, porque como defiende Stone Wiske (1999: 48-49): "Se trata de descubrir un tema, no de cubrirlo. La finalidad es la comprensión, no la acumulación de información aislada".

¿Por qué la contemporaneidad? La historia que se enseña y que se aprende se ha desarrollado tradicionalmente siguiendo un modelo cronológico clásico, es decir, un modelo basado en la sucesión, donde los acontecimientos históricos se ordenan siempre cronológicamente desde el pasado más alejado hasta la actualidad.

Esta forma de enseñar historia ha llevado a la distribución de los contenidos escolares en distintos temas o unidades, de manera que las realidades complejas del pasado se desmenuzan en múltiples partes para hacerlas más comprensivas. Parece una forma lógica de aprender, pero en realidad cada tema se desarrolla de una forma absolutamente cerrada, sin vínculos con los temas anteriores o posteriores, aunque correspondan a la misma unidad temporal.

De esta manera, como si se tratase de carpetas distintas de una computadora, se desarrollan y se evalúan siempre separadamente, sin buscar las posibles relaciones entre acontecimientos simultáneos o entre culturas o civilizaciones coetáneas.

Al respecto, Bellver (2001: 14) afirma: "Es relativamente sencillo enseñar por separado cada una de las partes que componen una estructura, pero es más complicado mostrar cómo cada una de éstas se armoniza con las otras para formar un conjunto". Cierto es que no se puede enseñar todo y todas partes, como cierto es que debemos seguir un orden determinado en la reconstrucción histórica, pero enseñar y aprender historia sin relacionar los distintos ámbitos de una realidad concreta (contemporaneidad interna) o sin establecer relaciones entre realidades coetáneas (contemporaneidad externa), sin duda, no facilita el desarrollo de una historia comprensiva sino que, contrariamente, supone una reconstrucción incompleta o sesgada del pasado, puesto que implica tan solo la utilización de algunas de las piezas del rompecabezas global y el olvido de otras tantas.

La contemporaneidad se vincula básicamente con la simultaneidad, pero en función de la unidad temporal utilizada también activa la aplicación 
de muchos otros operadores temporales como la sucesión, el cambio y la continuidad, la periodización, la coyuntura, la causalidad, y permite circular por el eje pasado-presente-futuro; en consecuencia, se trata de un operador temporal que fomenta la formación completa de la temporalidad en el alumnado, favorece la construcción de la conciencia histórica y, en última instancia, contribuye decididamente a la formación del pensamiento histórico en su conjunto y a la formación de una ciudadanía critica.

En este sentido, Santisteban y Pagès (2011: 230) afirman: "La formación de la temporalidad y la construcción de la conciencia histórica son objetivos fundamentales que cualquier ciudadano/a debe poseer para ubicarse en el mundo, comprender lo que está sucediendo e intentar cambiar aquello que no funciona y conservar lo que vale la pena". Pero la contemporaneidad también permite hacer visible al invisible, es decir, rompe con el eurocentrismo y etnocentrismo vigente para dar protagonismo a múltiples realidades, culturas o civilizaciones tradicionalmente obviadas.

Barton i Levstik (2000: 26) afirman: "Deberíamos ser capaces de pensar en los diferentes modos de incluir los diversos grupos en el currículo, de modo que no los posicione como enemigos". Efectivamente, si el enseñante de historia no hace visible a todos los estudiantes, difícilmente podrán sentirse que forman parte de una misma comunidad global, y es que, como defienden Oller y Pagès (1999: 7): "La sociedad del futuro no puede ser una suma de culturas, sino que debe fundamentarse en la construcción de proyectos comunes en los que todas las personas, autóctonas o inmigradas, participen en igualdad de condiciones".

La aplicación de la contemporaneidad en el aula ofrece pues múltiples oportunidades educativas y rompe con tendencias de herencia decimonónica que convierten la historia escolar en una historia anquilosada, básicamente memorística y muy poco funcional para nuestros chicos y chicas.

\section{Proceso de la investigación: Metodología}

Para poder confirmar las posibilidades reales de la aplicación de la contemporaneidad en las aulas de secundaria desarrollé un proceso de investigación-acción que, como estudio de caso, pretendía validar este modelo de enseñar historia. La investigación se desarrolló en el centro escolar, donde vengo desarrollando mi tarea docente por más de 20 años, FEDAC Sant Vicenç, un colegio cristiano concertado, de una sola línea de educación inicial y primaria y de dos líneas de Educación secundaria. Es un colegio situado en un pequeño pueblo de unos 9000 habitantes, 50 quilómetros al norte de Barcelona, con un alumnado muy heterogéneo y con un nivel sociocultural medio-bajo. 
A pesar de que realicé una primera parte de la investigación con el alumnado de Primero de la Enseñanza Secundaria Obligatoria (11-12 años), a partir de un cuestionario inicial y de dos focus-group, para conocer las concepciones temporales de estos chicos y chicas en el momento de iniciar esta etapa, el núcleo de la investigación se llevó a cabo durante todo el curso escolar 20122013 con el alumnado de cuarto de Secundaria (15-16 años). Este proceso consistía en determinar la situación inicial del alumnado en relación al dominio de la contemporaneidad y de los distintos operadores temporales vinculados al mismo, por lo que se aplicó un cuestionario inicial de raíz cualitativa, con preguntas que atendían a los diversos niveles de complejidad del operador a partir de recursos variados: imágenes, narraciones de vida, titulares de prensa.

Figura 1: Ejemplo cuestionario inicial pregunta 2

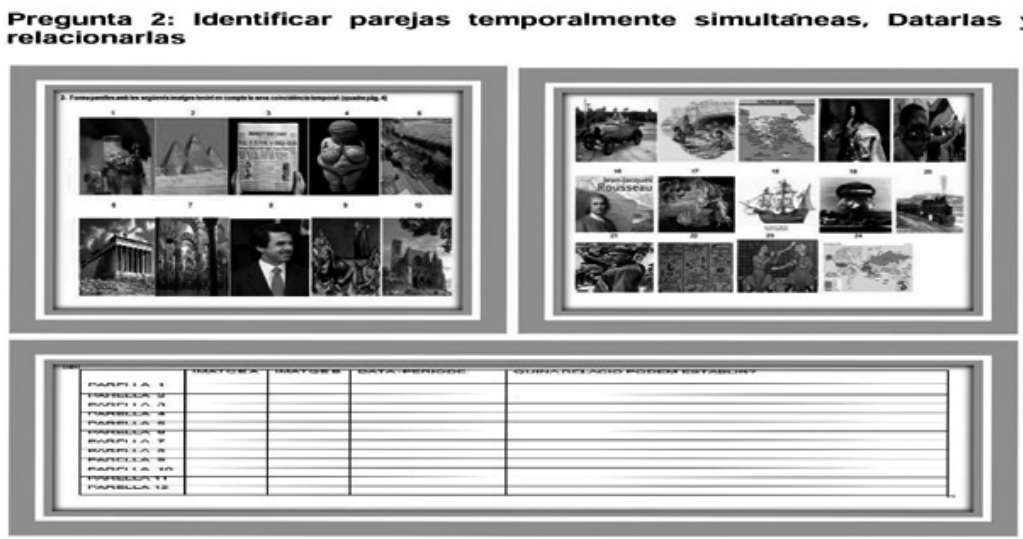

Fuente: Llusà, 2015

En una segunda fase del proceso de investigación, durante todo el curso escolar y siguiendo el currículum en vigor, se desarrolló en cada una de las unidades del curso el modelo de la contemporaneidad, prestando especial atención a tres intervenciones didácticas, una por trimestre, donde se pretendía obtener información específica sobre el proceso de construcción de la temporalidad.

Posteriormente, en los últimos días del curso escolar, se aplicó un nuevo cuestionario cualitativo al mismo alumnado, con ligeras modificaciones en relación al cuestionario inicial, de manera que permitiese la comparación entre ambos, pero no facilitase su realización por ser exactamente como el primero.

Finalmente, un año y medio después de desarrollar todo el proceso de innovación e investigación docente, se llevaron a cabo dos focus-group, absolutamente heterogéneos, con algunos de los alumnos que habían lle- 
vado a cabo este proceso en $4^{\circ}$ de Educación secundaria, con la intención de valorar la eficacia del modelo para el desarrollo de sus vidas cotidianas a nivel académico o profesional.

Se trató, por tanto, de un modelo de investigación-acción de didáctica de las ciencias sociales, que, siguiendo una tipología cualitativa y utilizando algunos instrumentos propios de la etnografía, permitiese interpretar una realidad específica, en este caso la de mi propia aula y de mi propio alumnado.

Figura 2: Proceso de investigación

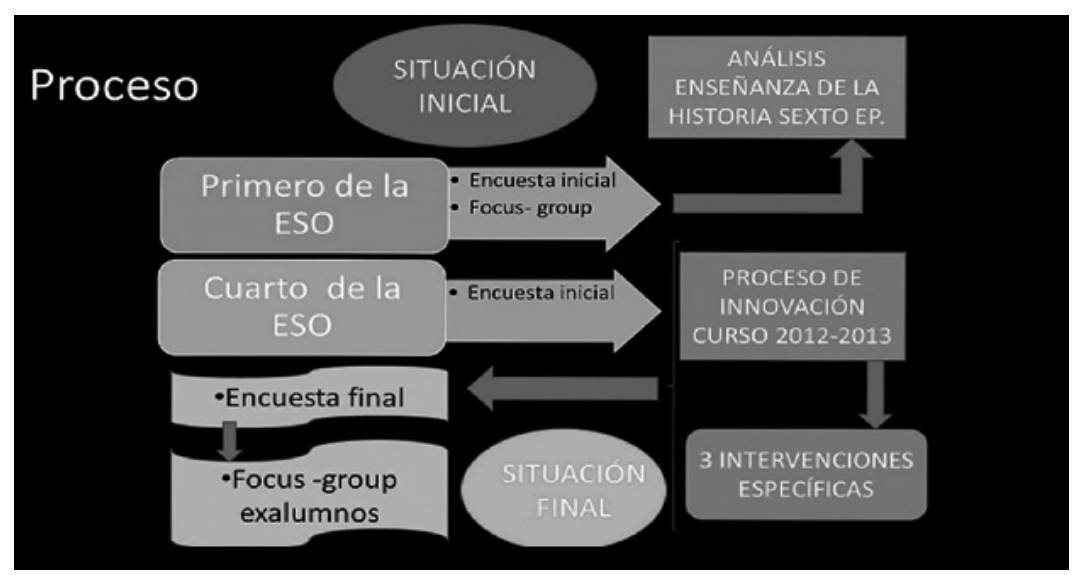

Fuente: Llusà, 2015

\section{Resultados de la investigación}

A pesar de tratarse de un estudio de caso, el desarrollo de un proceso de innovación e investigación en dos niveles distintos y a lo largo de un tiempo tan prolongado aportó un volumen ingente de información, tanto cuantitativa como cualitativa.

Para comprender mejor, tanto la construcción de la temporalidad en el alumnado, como la eficacia del modelo de enseñanza y aprendizaje de la historia basado en la contemporaneidad, presentaré específicamente los resultados de raíz cualitativa de la investigación, aunque puntualmente me referiré a algunos de los resultados cuantitativos de la misma.

\section{Primero de la ESO (Enseñanza Secundaria Obligatoria)}

En relación al alumnado de Primero de la ESO (Enseñanza Secundaria Obligatoria) -12 años-, a partir de los resultados del cuestionario y de los 
focus-group desarrollados, se puede afirmar que a la mayor parte les gusta aprender historia, aunque solamente le otorgan un valor de conocimiento del pasado y no la vinculan a la comprensión de su presente ni le dan ninguna funcionalidad o utilidad en sus vidas.

Explicitan haber desarrollado la asignatura de historia en educación primaria de una forma discontinua, incompleta y con una metodología claramente tradicional. El análisis de los manuales desarrollados por este alumnado y la entrevista con su profesora de ciencias sociales durante el último curso de primaria confirmaron estas afirmaciones.

La mayoría de los alumnos de primero de la ESO otorgan una gran importancia a la datación y a la cronología para poder ordenar los acontecimientos en el tiempo, pero en cambio, tan solo un 10\% es capaz de datar de forma precisa en el análisis histórico.

Cuando se les pide ordenar distintos hechos históricos, no contemplan la posibilidad de ordenarlos de forma diferente a la tradicional, es decir, de los más antiguos a los más recientes. Forma de ordenación lógica teniendo en cuenta que siempre han aprendido historia de forma cronológica y sucesiva, sin embargo, los chicos y chicas entrevistados, confirman la apreciación de Mattozzi (2002) en cuanto que confunden el orden del discurso con el orden temporal.

Esta idea me parece realmente preocupante, dado que la habitual ordenación cronológica clásica de los contenidos históricos, sin tener en cuenta la simultaneidad de los procesos históricos, de las relaciones existentes entre ellos o de los distintos ritmos de evolución de las diversas sociedades, no pueden hacer más que llevar a un aprendizaje de la historia claramente inconexo y superficial.

Estos chicos y chicas saben definir los distintos conceptos temporales como la duración, la sucesión o el cambio y permanencia, los saben ejemplificar y les atribuyen una importancia central para la comprensión histórica, pero muestran grandes dificultades de aplicación tanto en la interpretación como en la representación histórica. Saben que nuestro presente depende de los hechos desarrollados en el pasado y en consecuencia que de las acciones del presente depende el futuro, pero no parecen capaces de establecer una proyección lógica y coherente.

Santisteban (2009) defiende que el eje pasado-presente-futuro y el cambio y continuidad son los dos conceptos temporales más importantes para la formación de la estructura temporal. En ambos casos, los alumnos de primero de la ESO demuestran comprenderlos, pero no aplicarlos.

Esta misma afirmación nos sirve para el concepto de simultaneidad, puesto que entienden su significado e incluso ponen ejemplos de sus vidas o de su 
entorno más cercano, pero en cambio su aplicación al análisis histórico es casi inexistente. De esta forma podemos observar cómo en los distintos niveles de complejidad creciente del operador contemporaneidad en ningún caso el porcentaje de alumnos que lo aplican supera el $40 \%$.

Como vemos en la figura 3, tan solo un $30 \%$ del alumnado es capaz de identificar acontecimientos simultáneos y un 25\% de relacionarlos temporalmente, si bien llega al $40 \%$ el porcentaje de alumnos y alumnas que establecen relaciones no temporales (de ámbito, territoriales). La cifra aún es más limitada cuando se trata de explicar una realidad histórica a partir de estas relaciones, siendo inferior al 10\%, y prácticamente ningún chico o chica es capaz de aplicar el modelo de análisis de la contemporaneidad a otras realidades.

Figura 3: Aplicación porcentual de la datación y de los distintos niveles de contemporaneidad

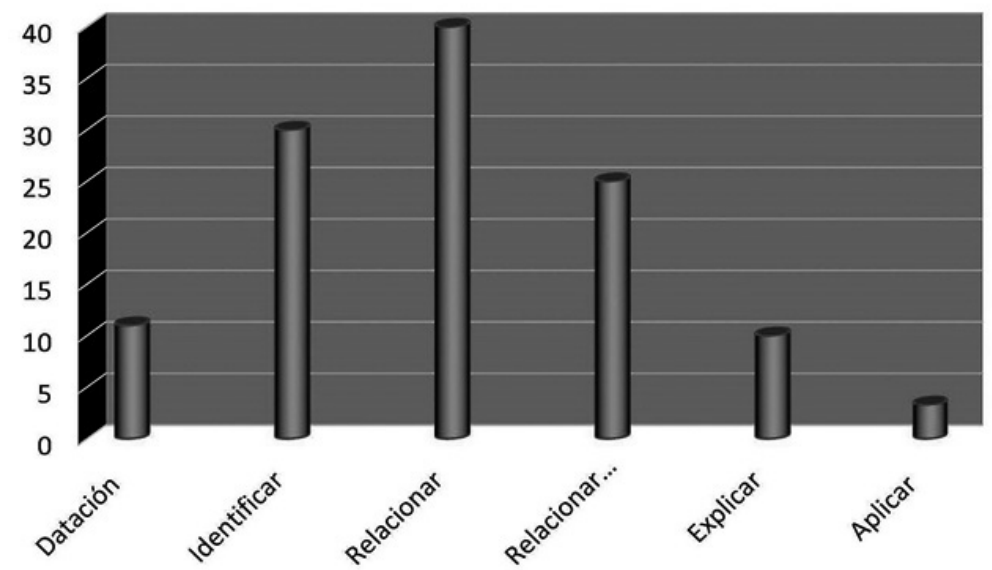

Fuente: Llusà, 2015

En relación a la aplicación a distintas realidades, se pone de relieve que la imagen de futuro es construida más por influencia de los medios de comunicación o el cine que con la prospectiva aplicada desde la lógica histórica. En este sentido se presenta un futuro altamente tecnificado o catastrófico, reproduciendo imágenes conocidas del cine o de los videojuegos y sin establecer relaciones con acontecimientos del presente o vinculaciones con el pasado.

En definitiva, el alumnado de 12 años del centro escolar FEDAC comprende la importancia de los distintos conceptos que articulan el tiempo histórico para la comprensión histórica, pero no dispone aún de los instrumentos necesarios o de la práctica adecuada para aplicarlos sistemáticamente al análisis histórico. 
Estos resultados ponen de manifiesto que el alumnado de esta edad, a pesar de no haber desarrollado ningún trabajo específico sobre tiempo histórico a lo largo de la educación primaria, es capaz de comprender el concepto y los operadores que lo articulan, por lo que el establecimiento de un currículum temporal paralelo al currículum establecido a lo largo de esa etapa y una adecuada secuenciación de sus conceptos articuladores, permitiría un mayor desarrollo cognitivo-temporal y una mayor facilidad de comprensión histórica.

En este sentido, Mattozzi (2002) plantea la educación temporal como centro de la educación histórica a partir de la interrelación entre los conceptos del tiempo y los conceptos históricos. Defiende que los conceptos temporales actúan como organizadores cognitivos, tanto en los acontecimientos de la vida cotidiana como en el proceso de comprensión del conocimiento histórico. Como él, múltiples autores: Pagès (1989, 1999), Guibert (1994), Bellver (2001), Prats (1997), Trepat (1998), Calvani (2005), Santisteban (2005), defienden esta posición.

Así, por ejemplo, Trepat afirma:

"Para un aprendizaje correcto del tiempo histórico no basta con la presentación una sola vez de los conceptos temporales y de sus operaciones. Es necesario, a lo largo de diferentes unidades y cursos a propósito de otros contenidos en los que intervengan las nociones temporales, ir explicando los mecanismos de cálculos, representación y periodización de manera constante, ampliando poco a poco su conceptualización" (1998: 51).

\section{Cuarto de la ESO}

Una vez conocida la situación de partida del alumnado al inicio de la educación secundaria, el proceso de investigación se centró en los chicos y chicas de $4^{\circ}$ de la ESO (15-16 años). Los resultados cualitativos obtenidos de los más de 50 alumnos y alumnas de este nivel al inicio del curso escolar demostraron, en general, un bajo nivel en el dominio de la contemporaneidad y en la aplicación de los distintos conceptos temporales investigados.

De los 57 alumnos y alumnas encuestados, más del 90\% no llegaron a obtener un puntaje suficiente. El alumnado del grupo 1 (chicos y chicas con un mejor rendimiento académico y que mayormente pretendían estudiar bachillerato y universidad posteriormente) obtuvo unos resultados muy superiores a los del grupo 2 (chicos y chicas que deseaban cursar formación profesional o incorporarse al mundo laboral), con un diferencial de casi 2 puntos sobre 10. Prácticamente el mismo diferencial podía apreciarse según 
el nivel sociocultural familiar, siendo muy superior el resultado en aquellos alumnos y alumnas con padres con mayor formación educativa, mientras que el diferencial según genero fue irrelevante.

En cualquier caso, el análisis cualitativo de la encuesta puso de relieve unos resultados preocupantes, con una progresión considerable, pero ciertamente limitada, respecto a lo acontecido en el $1^{\circ}$ de la ESO, como se puede observar en la figura 5.

Figura 4: Comparación porcentual datación y niveles contemporaneidad $1^{\circ}$ de la ESO y encuesta inicial de $4^{\circ}$ de la ESO

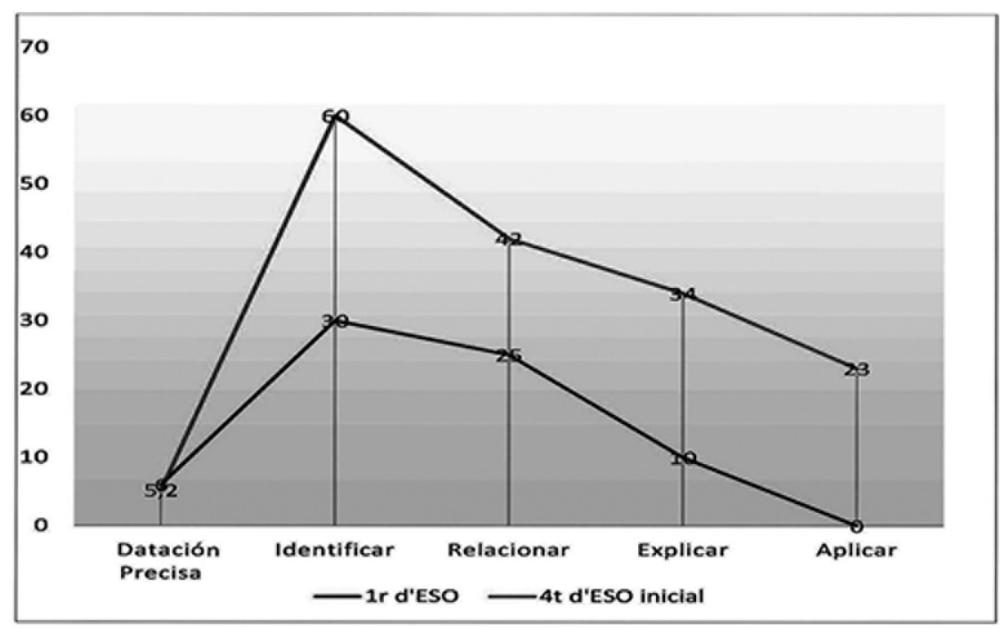

Fuente: Llusà, 2015

Como podemos observar la dificultad mostrada por el alumnado es directamente proporcional a la complejidad del nivel analizado; así podemos ver que, en relación a la capacidad de identificación de acontecimientos simultáneos, el $60 \%$ del alumnado de $4^{\circ}$ de la ESO es capaz de identificar acontecimientos simultáneos, especialmente si están vinculados a sus propias vidas o forman parte de la historia reciente, doblando en este caso a la capacidad demostrada por el alumnado de $1^{\circ}$ de la ESO.

Respecto al segundo nivel de complejidad, es decir, la capacidad de relacionar acontecimientos simultáneos temporalmente, el $40 \%$ de los chicos y chicas son capaces de establecer algún tipo de relación, siendo desarrollado tan solo por una cuarta parte del alumnado en el caso del alumnado de $1^{\circ}$ de la ESO.

En los dos últimos niveles de complejidad, es decir, la capacidad de utilizar las relaciones entre hechos simultáneos para explicar y la capacidad de aplicar 
este modelo de análisis histórico a cualquier realidad, podemos observar una dificultad creciente, siendo desarrollada tan solo por un tercio del alumnado en el primer caso y por menos de una cuarta parte en el segundo.

A pesar de estos datos, en relación al alumnado de $1^{\circ}$ de la ESO, podemos observar cómo la progresión es de 14 puntos en el tercer nivel y de 23 puntos en el cuarto, dado que los resultados obtenidos en ambos niveles por parte de los chicos y chicas de 12 años, denotaban una capacidad prácticamente inexistente.

En relación al dominio de los distintos conceptos temporales se ponen de manifiesto importantes dificultades, siendo especialmente destacadas en el caso de la datación precisa. En este mismo sentido Lautier (1997) también explicitaba las dificultades del alumnado de secundaria francés para dominarla adecuadamente.

Por lo que respecta al establecimiento de relaciones entre acontecimientos, mayoritariamente se establecen relaciones temáticas, territoriales o de ámbito, siendo las relaciones temporales las de menor aplicación y, entre éstas, las relaciones causales las más numerosas. El establecimiento de relaciones de simultaneidad es muy exiguo, siendo aplicado tan solo en 37 ocasiones entre todos los cuestionarios de todo el alumnado. Ante esta situación, como docente y como investigador, cabía actuar urgentemente si quería mejorar la temporalidad y la comprensión histórica de estos chicos y chicas antes de la finalización de su escolarización obligatoria.

Por esta razón, realicé durante todo el curso escolar 2012-2013 un proceso de innovación didáctica pensado especialmente para la consecución de este doble objetivo. Por ello, manteniendo el currículum en vigor y, por tanto, desarrollando los contenidos establecidos para este nivel educativo, llevé a cabo una adaptación metodológica en cada una de las unidades, con la intención de fomentar la comprensión y aplicación de los distintos conceptos temporales $y$, muy especialmente, aplicando de forma debidamente secuenciada los distintos niveles de complejidad creciente del operador contemporaneidad.

La observación participante se convirtió entonces en el instrumento fundamental de investigación y el diario de campo en elemento de sistematización de la misma.

A pesar de que la aplicación de la contemporaneidad estuvo presente en cada uno de los temas, elaboré tres intervenciones específicas, una por trimestre, con la intención de saber cómo se desarrollaba el proceso de construcción de la contemporaneidad.

En el primer caso, se realizó una intervención en grupo para potenciar la comprensión de la contemporaneidad interna, de forma que a partir de 
10 noticias de épocas y ámbitos distintos tenían que identificar 5 de ellas que fuesen simultáneas y que perteneciesen a la España del 2012. Una vez identificadas, debían añadir otras noticias simultáneas de cualquier realidad o ámbito para, posteriormente, relacionarlas entre sí con el objetivo de redactar un texto explicando la realidad del país en el presente. La actividad se desarrolló con mucha motivación y con unos muy buenos resultados.

En el segundo caso, en pequeños grupos prepararon una presentación en formato digital sobre un periodo de la historia de España del siglo XIX; una vez desarrollada, debían elaborar un friso cronológico completo del periodo (véase figura 2) y finalmente debían elegir una fecha o un pequeño periodo del friso para buscar qué sucedía simultáneamente en otros lugares del mundo. Una vez identificados los distintos acontecimientos debían buscar posibles relaciones entre ellos para explicar holísticamente qué sucedía en el mundo en la fecha o el periodo escogido. Esta intervención permitía potenciar tanto la contemporaneidad interna como la externa y, a su vez, comprender y aplicar múltiples operadores temporales.

Finalmente, la tercera intervención se vinculó a la historia retrospectiva, de manera que a partir de distintos gráficos de España 2000-2013 relativos a ámbitos distintos: política, economía, sociedad, cultura, tecnología, en pequeños grupos y de forma guiada, debían clasificarlos en la doble coyuntura: expansión (2000-2008) y crisis (2009-2013), para identificar, por tanto, la simultaneidad de acontecimientos. Posteriormente, debían describir cada gráfico para poder realizar posibles relaciones entre ellos y finalmente elaborar un texto explicando la situación global de España durante estos años.

Una vez desarrollada esta primera parte, a partir de gráficos y fuentes primarias correspondientes a la realidad norteamericana (1919-1939), los distintos grupos tenían que desarrollar una actividad como la primera, es decir, identificar aquellos gráficos y textos relativos a los "Felices años 20" y aquellos correspondientes a la "Depresión de los 30". Posteriormente debían relacionarlos para comprender ambas coyunturas y, finalmente, explicar globalmente la realidad norteamericana de entreguerras.

La parte final de la intervención consistía en desarrollar un video donde comparasen la realidad española del presente y la norteamericana de los años 20 y 30 del siglo pasado e hiciesen propuestas para solucionar la situación española actual. Las presentaciones se visualizaron en clase y finalmente se llevó a cabo un debate donde de forma argumentada se discutieron las soluciones propuestas por cada grupo.

Esta intervención fomentaba la contemporaneidad interna y externa a la vez, pero también permitía la comprensión y aplicación de distintos conceptos temporales como la simultaneidad, la causalidad, la sucesión, el ciclo y 
la coyuntura, el cambio y la continuidad, el eje pasado-presente-futuro, y es que como defiende Carrizo (2003: 7):

"Es indispensable que los alumnos analicen diferencias culturales -diferentes modos de vida y de solucionar conflictos - de sociedades muy distantes en tiempo y espacio. Para ello es necesario trabajar en la comparación contextualizada de distintas sociedades, y en la interrelación permanente de ida y vuelta entre el pasado y el presente, comprendidos en el estudio de los procesos históricos".

Después de desarrollar este largo proceso de innovación y de investigación durante todo un curso escolar, llegó el momento de comprobar la eficacia del modelo desarrollado. Para ello, se aplicó un nuevo cuestionario cualitativo, muy similar al primero, pero con cambios significativos en algunas preguntas, y se estableció, a partir del dominio de la contemporaneidad y de los distintos conceptos temporales, un sistema cuantitativo de comparación entre ambos.

En este sentido, se pudo apreciar una amplia mejoría en el dominio global de la contemporaneidad y de los conceptos temporales, de manera que prácticamente la mitad del alumnado fue capaz de completar adecuadamente el cuestionario, de manera que se produjo una variación positiva de casi el $40 \%$ en relación al cuestionario inicial.

Igualmente se produjo una progresión individual de más del $80 \%$ del alumnado, independientemente del grupo o del género.

Figura 6: Comparación cuantitativa contemporaneidad $4^{\circ}$ de la ESO entre encuesta inicial y encuesta final

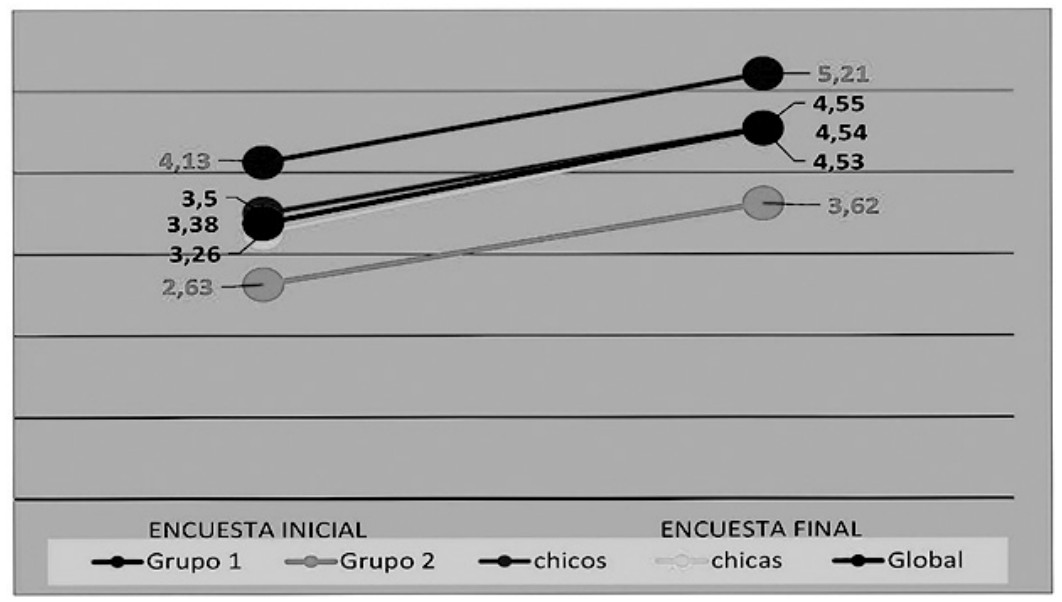

Fuente: Llusà, 2015 
Como podemos ver en el gráfico anterior, tanto el alumnado del grupo 1 como el del grupo 2, a pesar de que los primeros demuestran un nivel general superior, la progresión es de un punto sobre diez. Según género, en cambio, no podemos apreciar diferencias significativas, siendo en ambos casos una progresión similar.

El progreso desarrollado según nivel sociocultural familiar, a pesar de que el alumnado de un nivel alto partía de un puntaje muy superior, la progresión del alumnado con un nivel inferior es idéntica, siendo de prácticamente de 1,2 puntos sobre diez en ambos casos. Tan solo el alumnado con un nivel medio desarrolla una progresión menor, de alrededor de medio punto.

Estos resultados dan valor a la innovación desarrollada, puesto que, si bien no permiten reducir la distancia de partida en los resultados obtenidos según grupo de nivel o grupo sociocultural, sí permiten una progresión justa por parte de todo el alumnado.

Pero si los resultados cuantitativos pusieron de relieve un progreso muy importante por parte de prácticamente todo el alumnado, es en los resultados cualitativos donde se puede apreciar con toda su intensidad la validez del modelo didáctico desarrollado.

En este sentido se aprecia una progresión muy importante en relación a lo observado en $1^{\circ}$ de la ESO y en el cuestionario de inicio de $4^{\circ}$ curso, de manera que un $85 \%$ del alumnado demuestra saber identificar acontecimientos simultáneos, un $76 \%$ la capacidad de relacionarlos y un $43 \%$ de aplicar el modelo de análisis de la contemporaneidad a diversos contextos. De forma general, como podemos observar en la figura 7, la progresión se produce en más de un $20 \%$ en todos los niveles de complejidad del operador en relación a lo desarrollado en la encuesta inicial y de más de un $50 \%$ en relación a primero de la ESO.

Figura 7: Comparación porcentual niveles de contemporaneidad entre $1^{\circ}$ de la ESO y la encuesta inicial y la encuesta final de $4^{\circ}$ de la ESO.

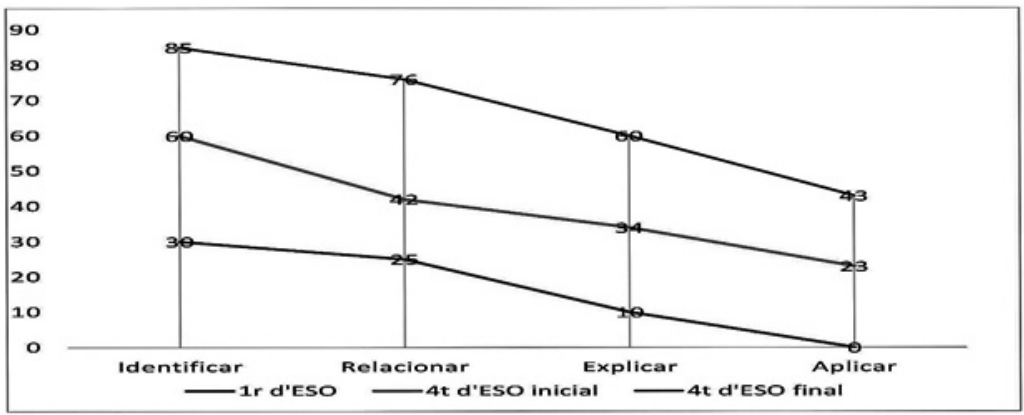

Fuente: Llusà, 2015 
En cuanto al uso de operadores temporales vinculados al desarrollo de la contemporaneidad, como podemos observar en la figura 8, respecto a la datación se produce una mejora muy considerable, siendo un 15\% más el alumnado capaz de datar y un $37 \%$ más de hacerlo de forma precisa. Sin embargo, esta progresión no esconde las dificultades del alumnado para datar, un problema común en el conjunto del alumnado de estas edades. Al respecto Lautier (1997: 21) afirmaba: "En histoire, les résultats révèlent une situation grave qui confirme que la chronologie est ignorée par la plupart des élèves de seconde et les sens chronologique absent".

En lo que se refiere a los otros operadores temporales, en la encuesta inicial se ponía en evidencia que solo una cuarta parte del alumnado los utilizaba para relacionar, mientras que en la encuesta final el porcentaje llegó casi al 60\%, lo que implica una mejoría de más de un tercio de los chicos y chicas. Más evidente es aún la mejoría en la utilización de estos operadores para relacionar, pasando de un $20 \%$ en el cuestionario inicial al $66 \%$ en el final. Entre estos operadores destaca especialmente la causalidad tanto en la inicial como en la final. La simultaneidad por su lado es el segundo operador más utilizado, aunque en su caso en la encuesta final se triplica su número de aplicaciones.

Por lo que se refiere al resto de operadores temporales, en el cuestionario inicial eran prácticamente inexistentes tanto para relacionar como para explicar; contrariamente, en el cuestionario final, la sucesión, el cambio y la permanencia, el periodo, el eje pasado-presente-futuro, aparecen ampliamente, de manera que, si en el cuestionario inicial los operadores temporales eran aplicados con una media de 0,5 por alumno/a, en la final la media pasa a 6,5 por alumno/a.

En el uso de operadores temporales para proyectar el futuro se aprecia también un progreso extraordinario, de forma que se pasa de un $30 \%$ del alumnado en la encuesta inicial a un $82 \%$ en la final.

Figura 8: Comparación porcentual en el uso de operadores temporales entre la encuesta inicial y la encuesta final de $4^{\circ}$ de la ESO

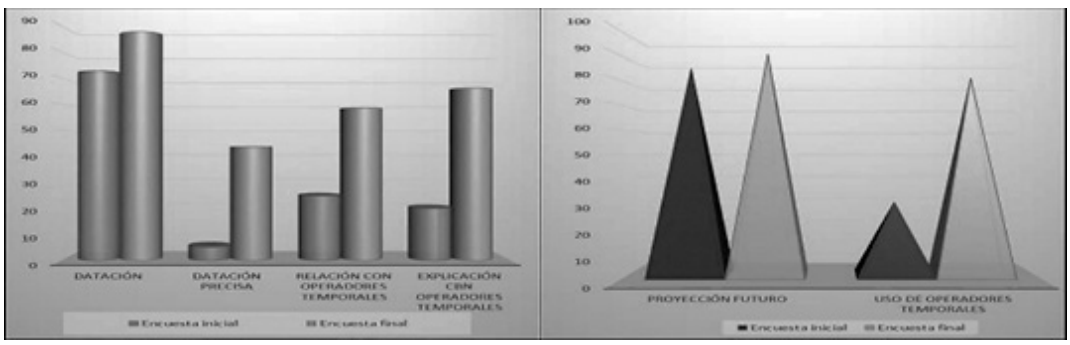

Fuente: Llusà, 2015 
Algunos autores como Carretero, Asensio y Pozo (1989: 115), defienden que: "Hasta los 15 o 16 años, los jóvenes solo demuestran tener visiones estáticas de la historia y una imagen de las diversas realidades del mundo, organizadas de forma estanca, sin conexión entre sí".

Pero los resultados obtenidos de ambas encuestas ponen de relieve que no es tan importante la edad del alumno como el trabajo específico en el aula basado en el dominio y la aplicación de los diversos conceptos articuladores de éste.

Parece evidente que la aplicación de la contemporaneidad en las aulas es un instrumento no solo útil, sino necesario para una correcta formación del pensamiento temporal del alumnado y, por extensión, de la comprensión histórica.

\section{Exalumnos}

Sin embargo, a pesar de esta evolución positiva en todos los aspectos, consideré necesario saber hasta qué punto el modelo aplicado de la contemporaneidad podía contribuir, no solo a la formación temporal del alumnado y a una mayor comprensión histórica, sino también a su funcionalidad social y a la construcción del pensamiento crítico y de la consciencia histórica, por ello, un año y medio después de finalizar el proceso de investigación e innovación en $4^{\circ}$ de la ESO, realicé dos focus-group con algunos de los alumnos y alumnas que lo habían desarrollado.

Estos chicos y chicas pusieron de relieve la importancia que tiene para ellos la historia, otorgándole un papel determinante, tanto en su vida académica como profesional. Para ellos la historia permite conocer el pasado, para interpretar el presente y proyectar el futuro. Valoran muy positivamente el proceso de innovación desarrollado en $4^{\circ}$ de la ESO, como un modelo comprensivo y no memorístico.

En su discurso se puede apreciar un importante dominio de los distintos operadores temporales, especialmente de la causalidad y de la simultaneidad, pero también del cambio y la permanencia. Así mismo demuestran un elevado nivel de comprensión, dominio y aplicación de los distintos niveles de complejidad de la contemporaneidad, de manera que saben identificar acontecimientos simultáneos y relacionarlos para explicar diversas realidades del pasado, del presente y del futuro.

En definitiva, estos exalumnos demuestran tener formada su consciencia histórica y desarrollado su pensamiento crítico, en tanto que son capaces de realizar una explicación crítica del presente a partir de ejemplos del pasado y poner de manifiesto su prospectiva de futuro. 
Resulta imposible cuantificar el grado de incidencia de la aplicación de la contemporaneidad en relación a la formación temporal de este alumnado y sería muy presuntuoso establecer una relación directa con la formación de su consciencia histórica y pensamiento crítico, pero ciertamente ha contribuido a ello.

Una de las chicas se expresaba de esta forma:

FA: "Ahora que te he podido comparar con otros profesores te puedo asegurar que tu método es mucho mejor que el tradicional porque nos haces vivir un momento como una unidad, que tenga un sentido, no un conjunto de fechas y de hechos. Esto hace que puedas establecer relaciones lógicas, recuerdes mejor y lo relaciones con el presente".

\section{Consideraciones finales}

En definitiva creo que este trabajo me permite demostrar que la aplicación de la contemporaneidad en el proceso de enseñanza y aprendizaje de la historia mejora la comprensión histórica del alumnado; que el proceso de construcción de la temporalidad se desarrolla especialmente durante la secundaria obligatoria, aunque empiece con anterioridad y se consolide posteriormente, y que la metodología del aprendizaje por descubrimiento guiado es óptima no solo para fomentar la construcción de la temporalidad, sino también para el aprendizaje de una historia más comprensiva.

Blythe afirma:

"Generalmente descubrimos que nuestros alumnos comprenden mucho menos de lo que habíamos esperado (...) tienen dificultades para escribir ensayos que muestren una auténtica comprensión del tema, más aún, no ven las conexiones entre lo comprendido en la escuela y sus actividades fuera de ella" (1999: 37).

Pero en cambio, esta forma de enseñar y aprender historia permite escapar de la enseñanza cronológica lineal, basada solo en la trama (la sucesión) y siempre presentada de forma aislada y compartimentada, para poner también la urdimbre de la historia y poder tejer conjuntamente la realidad compleja de la humanidad tanto del presente como del pasado.

Por ello, considero que la principal aportación de esta tesis doctoral es la de demostrar que se puede enseñar historia de una forma comprensiva y funcional a partir de la contemporaneidad, es decir, a partir de la identificación de hechos, culturas y civilizaciones desarrolladas en la misma unidad temporal, y establecer relaciones entre ellas para explicar holísticamente una realidad. 
Cierto es que se trata tan solo de una investigación basada en un estudio de caso y, por tanto, no pretende ser generalizable, pero sí permite por un lado evaluar mi propia docencia en pos de mejorarla y aportar a la investigación en didáctica de las ciencias sociales información relativa al proceso de construcción del conocimiento y del pensamiento temporal, y, por otro lado, servir de ejemplo a todos aquellos maestros y profesores que, como yo, creen firmemente que el cambio en educación es posible desde las aulas, porque yo como Gardner: "Quiero que mis hijos entiendan el mundo pero no solo porque el mundo sea fascinante y la mente humana rebose curiosidad. Quiero que lo entiendan para contribuir a hacer de él un hogar mejor" (2001: 181).

\section{Referencias bibliográficas}

\section{a) Artículos}

Barton, K.C.; Levstik, K (1996). "Back when God was around and everyting: Elementary children's understanding of historical time", en American Educational Research Journal, № 33, pp. 419-454.

Carrizo, K. (2003). "El concepto de simultaneidad en la enseñanza de la historia. Octavo año de $3 r$ ciclo de EGB", en Salta: Revista Escuela de Historia $N^{\circ} 2$. Facultad de Humanidades de Salta, pp. 1-8.

Oller, M. y Pagès, J. (1999). "La historia de los otros", en Revista Historia № 3 , pp. 172-187.

Pagès, J. (1989). "Aproximación a un currículum sobre el tiempo histórico", en Rodríguez Frutos, J. (Ed.). Enseñar historia. Nuevas propuestas. Barcelona: Laia, Cuadernos de pedagogía, pp. 107-138.

Pagès, J. (1999). "El tiempo histórico. ¿Qué sabemos sobre su enseñanza y su aprendizaje? Análisis y valoración de los resultados de algunas investigaciones", en AA. VV. Aspectos didácticos de Ciencias Sociales 13. (pp. 241-278). Zaragoza: ICE Universidad de Zaragoza.

Pagès, J. (2003). "Ciudadanía y enseñanza de la Historia" en Reseñas de Enseñanza de la Historia No 1, oct 2003, Revista de la APEHUN, As. de prof. de Enseñanza de la $\mathrm{H}^{\mathrm{a}}$. De Universidades Nacionales, pp. 11-42.

Tutiaux - Guillon, N. (2003). L'histoire enseignée entre coutume disciplinaire et formation de la conscience historique: I'exemple français». In Tutiaux -Guillon, N.; Nourrisson, C. (Coor.) Identités, mémoires, conscience historique. Saint-Étienne. Publications de l'Université de Saint-Étienne. 


\section{b) Libros}

Alonso Tapia, J. (Dir.) (1997). Evaluación del conocimiento y su adquisición. Volumen I Ciencias Sociales. Madrid: Ministerio de Educación y Ciencia/CIDE.

Asensio, M., Carretero, M., Pozo, I. (1989). "La comprensión del tiempo histórico" en: Carretero, M., Pozo, I., Asensio, M. (Comp.). La enseñanza de las Ciencias Sociales. Madrid: Aprendizaje Visor.

Bellver, A. (2001). El tiempo en la historia. Una metodología dinámica y activa en la ESO. Zaragoza: Edelvives.

Bloom, B. (1971). Taxonomía de los objetivos de la educación, la clasificación de las metas educacionales: manuales I y II. Buenos Aires: Centro Regional de Ayuda Técnica. Agencia para el Desarrollo Internacional (A.I.D.).

Blyte, T. y colab. (1999). La enseñanza para la comprensión. Guía para el docente. Barcelona: Paidós.

Calvani, A. (1988). Il bambino, il tempo, la storia. Firenze: La Nuova Italia.

Christian, D. (2005). Mapas del Tiempo. Introducción a la "Gran Historia". Barcelona: Crítica.

Gardner, H. (2001). La inteligencia reformada. Las inteligencias múltiples en el siglo XXI. Barcelona: Paidós.

Guibert, M. E. (1994). Tiempo y tiempo histórico. Navarra: Gobierno de Navarra. Departamento de Educación y cultura.

Lautier, N. (1997). Enseigner I'histoire au lycée. Paris: Armand Colin.

Mattozzi, I. (2002). Presentazione per il docente. La formacione del pensiero temporale negli adolescenti. En: Perillo, E. (a cura di). La Storia. Istruzione per I'uso. Materil per la formazione de competenze temporali degli studenti. Napoli: Tecnodid.

Mcneill, J. R.; Mcneill, W. H. (2004). Las Redes humanas, una historia global del mundo. Barcelona: Crítica.

Prats, J. (1997). La investigación en Didáctica de las Ciencias Sociales (notas para un debate deseable). AUPDCS (ed). La formación del profesorado de Didáctica de las Ciencias Sociales. Sevilla: Díada.

Santisteban, A. y Pagès, J. (2011). Enseñar y aprender el tiempo histórico. Madrid: Síntesis.

Sthenhouse, L. (1998). La investigación como base de la enseñanza. Madrid: Morata. 
Stone Wiske, M. (1999) (comp). La enseñanza para la comprensión. Vinculación entre la investigación y la práctica. Barcelona: Paidós.

Trepat, C. A.; Comes, P. (1998). El tiempo y el espacio en la didáctica de las ciencias sociales. Barcelona: ICE/UB-Graó.

c) Tesis

Llusà Serra, J. (2015). Enseñar historia desde la contemporaneidad. Estudio de caso sobre la construcción y aplicación del concepto de contemporaneidad en la ESO. Barcelona: Tesis doctoral, Universidad Autónoma de Barcelona.

Santisteban, A. (2005). Les representacions i l'ensenyament del temps històric. Estudis de cas en formació inicial de mestres de primària en Didàctica de les Ciències Socials. Barcelona: Tesis doctoral, Universidad Autónoma de Barcelona. 